\title{
L'utilisation d'Internet pour la démonstration et la simulation de techniques utilisées en télécommunications : de Fourier à l'ADSL.
}

\author{
Yves MOREAU*, Jérôme GALY** \\ *Centre d'Electronique et de Micro-Optoélectronique, Courrier 084 -Université Montpellier II \\ ** Laboratoire d'Informatique et de Robotique de Montpellier \\ Université Montpellier II -34 095 - Montpellier cedex 5
}

\begin{abstract}
Résumé : Nous présentons ici une utilisation encore peu fréquente dans le domaine des sciences, de ce que les outils internets peuvent offrir : au-delà du simple affichage de documents, la présentation active de processus scientifiques, ainsi que la modélisation. Les simulations peuvent être effectuées en ligne par l'utilisation de logiciels de navigation dotés de machines virtuelles (tels que les logiciels usuels Netscape ou Internet Explorer).
\end{abstract}

Mots clés : Internet, simulations, applet java, 'TICE', Fourier, ADSL.

\section{Introduction}

Nous avons développé des applets Java, utilisées pour certaines, comme démonstrationexpérience en support de cours, et pour d'autres comme expérimentations virtuelles. Leur implantation sur le réseau permet aussi aux étudiants ou internautes intéressés, de les reprendre dans d'autres environnements. A ce jour, sont accessibles outre la classique synthèse de Fourier (avec support auditif!), la convolution, le filtrage par ondelettes, la technique ADSL, le calcul des modes optiques, la propagation guidée en optique intégrée (plusieurs méthodes), l'accès multiple par code (CDMA) utilisée en téléphonie troisième génération.

Les démonstrations à l'aide d'applets Java constituent un outil adapté qui complète les explications orales de l'enseignant lors des cours. Les explications peuvent être aussi reportées sur des pages HTML pour former un cours complet à distance !

Le paragraphe $\underline{2}$ donne quelques éléments sur les applets java. Nous décrivons au paragraphe $\underline{3}$, trois applets : de Fourier à l'ADSL que l'on peut pédagogiquement enchaîner. La connexion ADSL (Asymetric Digital Subscriber Line) y est présentée sous son aspect modulation multiporteuse perturbée par un bruit non blanc. Nous citons brièvement au paragraphe 4 d'autres applications développées dans un but pédagogique.

\section{Applets Java}

L'explosion d'Internet s'est accompagnée non seulement d'outils permettant l'affichage passif de textes ou de dessins mais aussi d'outils actifs. La société Sun Microsystems a conçu une technologie : les 'applets Java' qui sont de réelles applications (programmes) exécutées sur l'ordinateur client (local) de façon transparente pour l'utilisateur. Les animations par exemple, sont calculées localement plutôt que transmises, ceci allège considérablement la quantité de données en transit sur le réseau. Le calcul local rend aussi plus souple la gestion de base de données, et bien sur les calculs. Il existe d'autres outils du même type php, le javascript, les programmes cgi, nous renvoyons le lecteur intéressé aux nombreux ouvrages ou site 'web' spécialisés.

Le langage évolué utilisé pour l'écriture d'applets Java [1] est orienté objet, il reprend pratiquement les possibilités et les avantages $\mathrm{du} \mathrm{C}++$ et permet de façon analogue à celui-ci le calcul scientifique avec résolutions numériques d'équations etc. Java intègre aussi les outils d'interfaçage homme-machine traditionnels tels que fenêtres, boutons, menus. Le chargement du code compilé java ("applet") est la seule information transmise depuis le site (de l'ordre de quelques dizaines de kilo-octets). 
L'interprétation du code et l'exécution du programme sont effectuées localement de façon transparente sur l'ordinateur de l'utilisateur consultant le site à l'aide de son navigateur internet (Netscape, Internet explorer, Opera, Mozilla...)

Si les applications à base d'animations et/ou de consultations de bases de données sont nombreuses, il existe à notre connaissance peu d'applications Java tournées vers le calcul scientifique. L'intérêt pédagogique est lié à quatre aspects :

- programmes accessibles par Internet, outil attractif, apprécié et utilisé par les étudiants,

- aspect visuel bien développé, lié à la multiplicité des classes d'objets inclus dans l'environnement Java,

- intégration directe des explications scientifiques : une applet java est habituellement lancée depuis une page d'hypertexte («HTML ») pleinement adaptée aux explications hiérarchisées et aux liens vers d'autres rubriques,

- utilisation gratuite.

D'autres avantages peuvent être mentionnés comme la mise à jour aisée des programmes, et la possibilité d'une exécution sur des systèmes d'exploitations différents (Windows, Linux, etc.)

La rapidité d'exécution est maintenant voisine de celle de programmes écrits en langage $\mathrm{C}$ ou $\mathrm{C}++$, essentiellement depuis l'apparition de nouvelles techniques logicielles: compilation du code à la première utilisation lors d'une session (compilation Just In Time).

\section{De Fourier à l'ADSL}

Des logiciels ont été développés pour expliquer visuellement les notions nécessaires à la compréhension des techniques modernes utilisées en télécommunications. L'un de ceuxci [2], présente une "machine hydraulique" faisant varier sinusoidalement le niveau d'un premier réservoir, voir figure 1. Un deuxième réservoir communique avec le premier par un tuyau à débit limité, son niveau tente de suivre le premier.

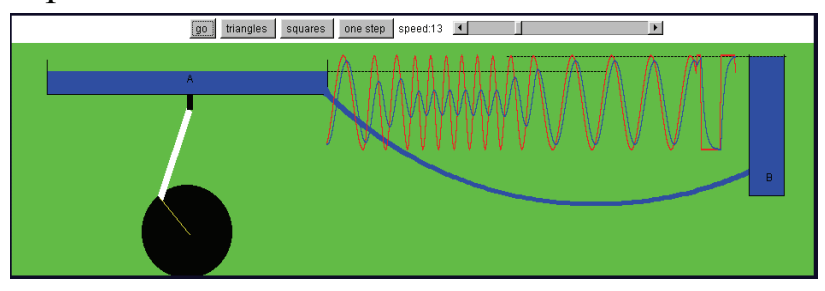

Figure 1: Machine -filtre passe-bas

On peut observer que les variations de niveau du second réservoir n'ont pas la même amplitude, et ceci d'autant plus que le mouvement est rapide (la vitesse est ajustable). De plus un retard peut apparaître.

On pourra par exemple tracer le diagramme de Bode pour cette machine en comprenant concrètement pourquoi l'amplitude est limitée à haute fréquence (très courant dans la nature), ou pourquoi un retard apparaît dans le mouvement élémentaire sinusoïdal, et comment ceci se traduit pour un signal plus complexe : signal carré ou signal triangulaire que l'on peut aussi expérimenter.

Une autre simulation (beaucoup plus difficile du point de vue calcul) est effectuée de façon implicite: Le profil du tuyau suit le mouvement des réservoirs, il est régi par une équation différentielle, qui régit aussi la courbe décrite par des fils électriques entre deux poteaux..

Cette applet devrait faire comprendre le mécanisme du filtrage passe-bas grâce à des phénomènes plus concrets que ce que l'on connaît en électronique du type charge et décharge de condensateur. On reprend ici l'analogie que les physiciens du dix-neuvième siècle utilisaient vraisemblablement, d'après les termes qu'ils ont choisis : résistance, capacité, courant...

La traditionnelle décomposition et synthèse de Fourier a été reprise, agrémentée dans notre applet [3] d'une synthèse sonore illustrant ici aussi la terminologie : harmoniques, octave etc. L'utilisateur peut lui-même composer les harmoniques de façon à reconstituer un signal donné, ou demander à l'ordinateur de les 
calculer. Il peut les modifier, les supprimer et les entendre...La présentation choisie est celle des électroniciens (amplitude et phase), plutôt que celle des mathématiciens (cosinus et sinus ou parties réelles et imaginaires).

Un utilisateur peut effectuer des simulations de façon très simple, sans "l'intendance" que réclame l'utilisation d'un logiciel de calcul scientifique.

Après une familiarisation avec la décomposition et la synthèse Fourier [3]], l'étudiant pourra expérimenter quelques techniques modernes de télécommunications. Là encore les 'applets' peuvent offrir une démonstration interactive.

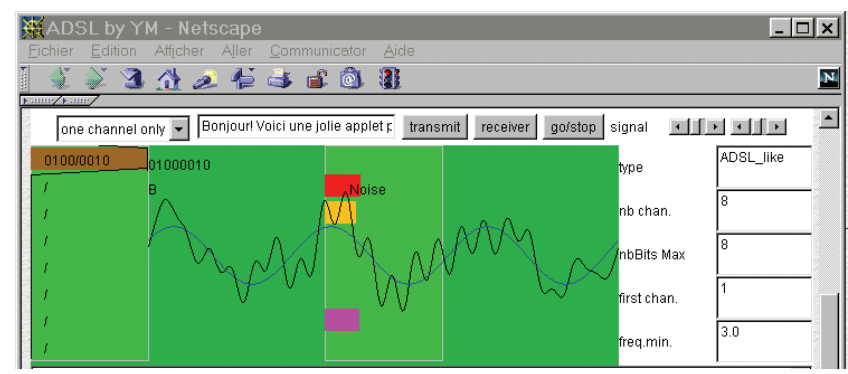

Figure 2: Fenêtre d'émission, signal sur un seul canal avec et sans bruit (interférences) sur d'autres canaux.

Nous ne ferons qu'évoquer ici les aspects traités sur notre site, en particulier sur la page 'ADSL' (Asymetric Digital Subscriber Line: technique utilisant pleinement la bande passante offerte par la liaison filaire qui relie le central et l'abonné au téléphone). La figure 2 montre la génération d'un signal QAM (modulation en quadrature) construit par l'applet [4] dans la fenêtre d'un navigateur (Netscape): un texte quelconque de l'utilisateur est codé en ASCII: les quatre premiers bits sont traduits en un niveau d'amplitude (parmi seize) d'une cosinusoïde tandis que les quatre bits suivants modulent une sinusoïde. On peut noter à cette occasion que les cosinus et sinus ne sont pas porteurs d'information en même temps au cours de la période, ce qui permet de transmettre deux informations en partage temporel implicite. Cette technique de duplexage est largement utilisée: système NTSC et PAL en télévision couleur.

La fenêtre de réception fait apparaître le décodage en sortie : amplitude de la cosinusoide et de la sinusoide, puissance spectrale, ainsi que les bits restitués et le texte. On peut expérimenter l'effet d'un bruit (typiquement une interférence) sur le signal et sur la réception selon l'intensité et la fréquence de l'interférence.

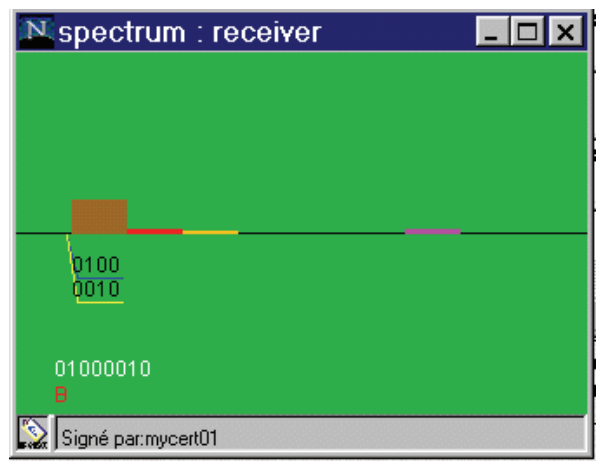

Figure 3: Fenêtre de réception : cas d'un canal, où chaque groupe de quatre bits correspond à une amplitude soit du cosinus, soit du sinus.

La démonstration peut continuer avec quelque huit canaux en parallèle (ajustable) caractérisés chacun par une fréquence porteuse multiple d'une fréquence de base (ADSL:4,125 $\mathrm{kHz}$, les premiers multiples n'étant pas utilisés dans la norme pour laisser libre la bande téléphonique usuelle).

Le signal ADSL est affiché (figure 4 lors de sa génération par modulation d'amplitude en quadrature et sommation sur tous les canaux.

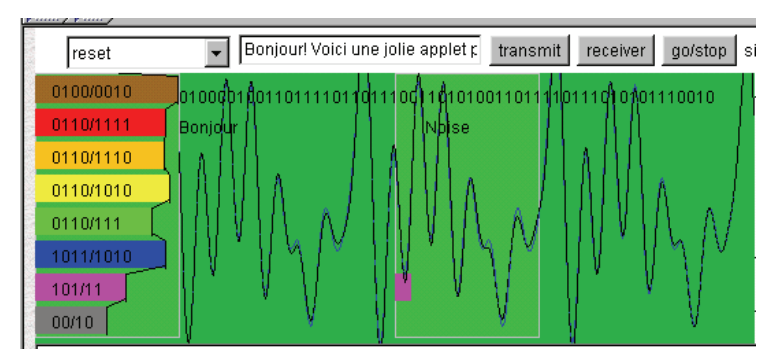

Figure 4. Signal ADSL avec huit sous-bandes dont la septième est bruitée.

La fenêtre de réception (fig.5) analyse le signal bruité transmis et reconstitue le flot de bits le texte, par une analyse parallèle sur tous 
les canaux (transformée de Fourier rapide). L'expérimentateur peut introduire (à l'aide la souris) du bruit sur certains canaux (simulant une perturbation) entraînant quelques erreurs au niveau de la réception. L'expérimentateur peut réduire (à la souris) le nombre de bits transmis sur le canal perturbé pour restaurer une transmission sans erreur (au prix d'une réduction du nombre de bits transmis, donc d'un débit binaire plus faible).

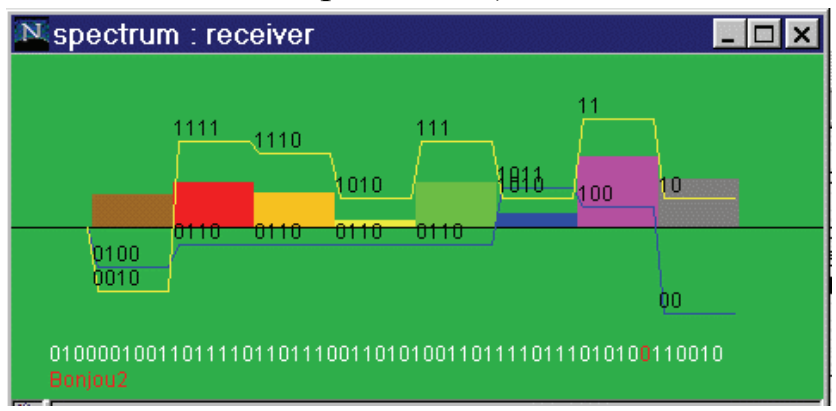

Figure 5. Réception d'un signal ADSL : une erreur sur un canal, peut être annihilée par une réduction du nombre de bits transmis sur ce canal.

Cette applet est utilisée en "expérience de cours" en premier cycle et troisième cycle, à différents niveaux d'approfondissement.

\section{Autres applets}

D'autres simulations sont accessibles sur le site Internet pour la présentation d'autres techniques, pour l'expérimentation pratique, ou encore pour des calculs plus complexes utilisés en recherche [5]. Nous avons développé des applets montrant la compression par ondelettes, calculant les modes optiques, leur propagation, présentées précédemment [6][7]. Celles-ci sont utilisées à l'IUP Optoélectronique. D'autres logiciels sont développés ou en cours de développement pour l'enseignement du traitement du signal (la convolution) ou d'autres techniques de télécommunications (l'accès multiple C.D.M.A)

La maturation du logiciel oblige aussi l'enseignant à réfléchir sur la communication pédagogique à effectuer, probablement beaucoup plus que lors de la préparation d'un enseignement traditionnel.
Nous démontrons aussi une méthodologie de programmation fondée sur l'approche objet qui convient au développement relativement rapide de telles applications : réutilisation de "méthodes" adaptées au calcul scientifique et à leur présentation... Chaque problème est caractérisé par une boucle infinie où après l'initialisation d'un ensemble de données, l'applet lance un calcul, affiche les conséquences du calcul, attend la modification de données...La classe problème ou Data peut rassembler un grand nombre de méthodes réutilisables sur l'acquisition de paramètres le lancement d'un "thrace d'animation" etc.

\section{Conclusion}

L'intérêt pédagogique de la simulation et de la visualisation grâce à des applets est mis en évidence, il est lié à de nombreux avantages :

- éventail d'applications large,

- aspect visuel important,

- rapidité de l'exécution, voisine de programmes écrits en $\mathrm{C}$ depuis l'apparition de la compilation "Juste In Time".

- Simulations accessibles par Internet, outil attractif, apprécié et utilisé par les étudiants.

Nous ne saurions trop encourager les enseignants à développer ce type d'approche et les institutions à les y inciter.

\section{Références}

[1] http://www.javasoft.com

[2] http://www.cem2.univ-montp2.fr/ moreau/machine/

[3] http://www.cem2.univ-montp2.fr./ moreau/fourier/

[4] http://www.cem2.univ-montp2.fr/ moreau/adsl/

[5] Y.Moreau, J.Porque, P. Coudray, P.Etienne, R.Kribich,Int'l Conf. SPIE, 3780, Denver, USA, (1999).

[6] Y.Moreau, R.Kribich, P. Coudray, Colloque CETSIS-EEA'99, 229-232, Montpellier (1999).

[7] Y.Moreau, R.Kribich, P. Coudray, JET'99, invited paper, 183-188, Nice-Sophia-Antipolis, (1999). 